\title{
Endophytes of the tall fescue ploidy series in Europe, North Africa and the Mediterranean
}

\author{
C.L. SCHARDL ${ }^{1}$, K.D. CRAVEN ${ }^{2}$, K.K. SCHWERI ${ }^{1}$, W. HOLLIN ${ }^{1}$, S.L. CLEMENT ${ }^{3}$, J. SCHMID ${ }^{4}$, C.P. WEST ${ }^{5}$, and T.D. PHILLIPS ${ }^{6}$ \\ ${ }^{1}$ University of Kentucky, Department of Plant Pathology, 201 PSB, 1405 Veterans Dr., Lexington, KY 40546-0312, U.S.A. \\ ${ }^{2}$ The Samuel Roberts Noble Foundation, Plant Biology Division, 2510 Sam Noble Parkway, Ardmore, Oklahoma 73401 \\ ${ }^{3}$ U.S. Department of Agriculture Agricultural Research Service (USDA-ARS), Washington State University, 59 Johnson Hall, \\ Pullman, WA 99164-6402, U.S.A. \\ ${ }^{4}$ Massey University, Institute of Molecular Science, Tower D, Riddet Road, Palmerston North, 5331 New Zealand \\ ${ }^{5}$ University of Arkansas, Department of Agronomy, ALTH \#224, Fayetteville, AR 72704-6898 \\ ${ }^{6}$ University of Kentucky, Department of Plant and Soil Sciences, 325 PSB, 1405 Veterans Dr., Lexington, KY 40546-0312, U.S.A.
}

schardl@uky.edu

We investigated on the genetic diversity of endophytes in native populations of tetraploid, hexaploid and octaploid tall fescue (Lolium arundinaceum) in Europe and North Africa. In hexaploid tall fescue, different species of endophytes had previously been identified and designated FaTG-1 (= Neotyphodium coenophialum), FaTG-2 and FaTG-3. We investigated the possibility that hosts of the different Neotyphodium species represented cryptic species of hexaploid tall fescue. Hosts of $N$. coenophialum were identified in northern Europe, Iberia, and Morocco, but not elsewhere in northern Africa. Hosts of FaTG-2 and FaTG-3 were identified in the southern European peninsula of Iberia and island of Sardinia, as well as northern Africa. Chloroplast sequence phylogenies suggested that hosts of FaTG2 and FaTG-3 were closely related to each other, but as distantly related to hosts of $N$. coenophialum as they were to Lolium multiflorum (an annual ryegrass), L. perenne (perennial ryegrass) and L. pratense (meadow fescue). Genome sizes of both European and Mediterranean tall fescue were estimated by staining nuclei with CyStain ${ }^{\circledR}$ UV Precise P and assessing fluorescence with a Partec Ploidy Analyser PA-I. Hosts of N. coenophialum had significantly larger genomes than hosts of FaTG-2 and FaTG3 (Fig.1). These results support the hypothesis that hexaploid tall fescue comprises two or more cryptic species with partially overlapping ranges, and that the different cryptic species host different endophyte species. Interestingly, $N$. coenophialum was also identified in tetraploid tall fescue from France (near Apt) and octaploid tall fescue from Morocco. Its occurrence in the tetraploid is in keeping with published chloroplast phylogenies, suggesting that $N$. coenophialum was in the tetraploid maternal ancestor when it hybridised with meadow fescue to give the more common hexaploid variety.

Keywords: Clavicipitaceae, epichloë endophytes, Festuca arundinacea, genome size, grasses, host specificity, Lolium arundinaceum, molecular phylogenetics, Neotyphodium, Poaceae, Pooideae, Schedonorus phoenix

Figure 1 Relative genome sizes of hexaploid plants with endophytes Neotyphodium coenophialum, FaTG2 or FaTG3. Genome sizes are based on comparisons with plant 15, which was set to 6.00 units. Plant designations are listed, followed by endophyte species if identified by DNA sequence. Otherwise, endophyte spore morphology is indicated as short-spored $\left({ }^{\star}\right)$, consistent with FaTG2 or FaTG3, or long spored (†), consistent with N. coenophialum.

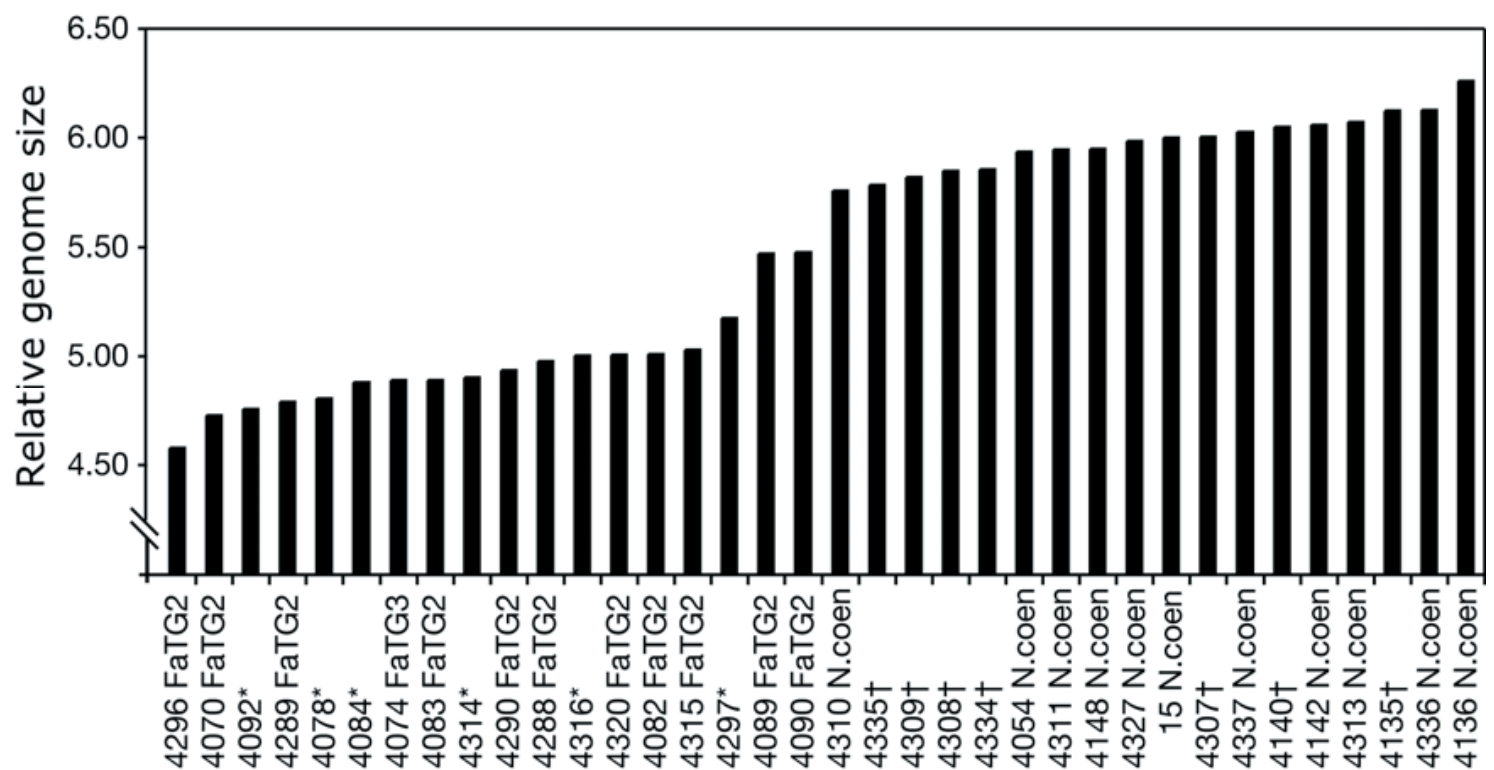

